\title{
Beneficial effects of tripterygium glycosides tablet on biomarkers in patients with ankylosing spondylitis
}

\author{
WEI JI $^{1}$, YAJUN CHEN ${ }^{1}$, XIA ZHAO ${ }^{1}$, YUNKE GUO ${ }^{1}$, LINGYU ZHONG ${ }^{1}$, \\ HONGGANG $\mathrm{LI}^{2}$, DAN WANG ${ }^{3}$ and YANNA SONG \\ ${ }^{1}$ Department of Rheumatology, Affiliated Hospital of Nanjing University of Traditional Chinese Medicine, \\ Nanjing, Jiangsu 210029; ${ }^{2}$ Department of Rheumatology and Immunology, Zhuzhou City Hospital, \\ Zhuzhou, Hunan 412000; ${ }^{3}$ Department of Tuberculosis, Nanjing Chest Hospital, Nanjing, Jiangsu 210029; \\ ${ }^{4}$ Department of Immunity, Nanjing Jiangbei People's Hospital, Nanjing, Jiangsu 210048, P.R. China
}

Received February 14, 2014; Accepted November 19, 2014

DOI: $10.3892 / \mathrm{mmr} .2015 .3448$

\begin{abstract}
The aim of the current study was to explore the effects and possible mechanisms of tripterygium glycosides tablet (TGT) in the treatment of active ankylosing spondylitis (AS). Thirty-six patients with active AS were given a $20 \mathrm{mg}$ TGT treatment three times per day for 12 weeks, and 21 unrelated healthy controls were recruited as the control group. Efficacy measures included the Bath AS disease activity index (BASDAI), erythrocyte sedimentation rate (ESR) and C-reactive protein (CRP) prior and subsequent to TGT treatment. Serum dickkopf homolog 1 (DKK1) and interleukin-17 (IL-17) levels before and after TGT treatment were assessed using reverse transcription-quantitative polymerase chain reaction (RT-qPCR) and ELISA assay. The levels of several serum biomarkers were determined by ELISA, including receptor activator of nuclear factor $\kappa$-B ligand (RANKL), osteoprotegerin (OPG), bone alkaline phosphatase (BAP), bone morphogenetic protein-2 (BMP-2), matrix metalloproteinase-3 (MMP-3), cross-linked telopeptide of type II collagen (CTX-II), vascular endothelial growth factor (VEGF), and prostaglandin E2 (PGE2). After 12 weeks of TGT treatment, the BASDAI score of the patients was significantly reduced $(\mathrm{P}<0.05)$, their levels of ESR and CRP were significantly reduced to a normal level $(\mathrm{P}<0.05, \mathrm{P}<0.05)$, RT-PCR and ELISA showed a significant increase in the level of DKK1 expression $(\mathrm{P}<0.05)$ and a significant decreased IL-17 expression $(\mathrm{P}<0.05)$, there was a significant increase in the expression of OPG, BAP and BMP-2 $(\mathrm{P}<0.01, \mathrm{P}<0.01$, $\mathrm{P}<0.01)$ and a significant reduction in the expression levels of RANKL, CTX-II. MMP-3, PGE2, and VEGF ( $\mathrm{P}<0.01, \mathrm{P}<0.01$,
\end{abstract}

Correspondence to: Dr Wei Ji, Department of Rheumatology, Affiliated Hospital of Nanjing University of Traditional Chinese Medicine, 155 Hanzhong Road, Nanjing, Jiangsu 210029, P.R. China E-mail: weiweiji1103@163.com

Key words: tripterygium glycosides tablet, ankylosing spondylitis, biomarker, anti-inflammatory effect, new bone formation
$\mathrm{P}<0.01, \mathrm{P}<0.05, \mathrm{P}<0.01)$ compared with those of the controls. TGT is effective at improving the signs and symptoms of patients with AS through the regulation of serum biomarkers, and the mechanisms may be associated with the anti-inflammatory effect, inhibition of new bone formation and potential bone-protective effects.

\section{Introduction}

Ankylosing spondylitis (AS) is a chronic inflammatory disease that primarily affects the axial skeleton, peripheral joints and the attachment of ligaments and entheses. The predominant clinical feature of this disease is inflammatory lower back pain, and over time certain patients develop spinal immobility and ankylosis (1,2). AS occurs predominately in males (3). The prevalence of AS is $0.2-0.54 \%$ among the Han Chinese population, which accounts for $92 \%$ of the whole Chinese population (4-6),with similar rate of prevalence in Europe and America. The clinical manifestations, severity, and risk of developing the disease vary by ethnicity, geography and gender, with a prevalence that is higher in young males and lower in certain populations, such as Japan $(7,8)$.

Despite a long standing knowledge of the familial associations of AS, the fundamental pathogenetic mechanism remains undefined. A number of cytokines have been shown to have critical roles in the pathogenesis of AS, including inflammatory cytokines and bone metabolism-related factors. Interleukin (IL)-17 and IL-23 are cytokines associated with inflammation, autoimmunity and defense against bacteria. The elevated levels of IL-17 and IL-23 in patients with AS indicates that they have critical roles in the pathogenesis of AS $(9,10)$. Vascular endothelial growth factor (VEGF) has a crucial role in angiogenesis, which is important for the pathogenesis of chronic inflammatory diseases in joints. Angiogenesis is a potential novel target for therapeutic intervention in inflammatory joint disease. The disease status of AS has been shown to be associated with elevated VEGF plasma levels (11). Prostaglandin E2 (PGE2) is an important factor for osteoblast differentiation in AS. PGE2 has anabolic effects on bones and promotes proliferation and differentiation of osteoblasts, thereby inducing the expression of bone sialoprotein and bone 
alkaline phosphatase (BAP) (12). The above inflammatory factors are closely associated with the pathogenesis and prognosis of AS.

In addition, numerous bone metabolism-associated factors are involved in AS. DKK-1 is an inhibitory molecule that regulates the Wnt pathway, which controls osteoblastogenesis, and the potential role of DKK-1 in AS has been explored (13). Besides, a number of other bone metabolism-associated factors are involved in the formation of AS, including the receptor activator of nuclear factor $\kappa \mathrm{B}$ ligand (RANKL), osteoprotegerin (OPG), bone alkaline phosphatase (BAP) and bone morphogenetic protein-2 (BMP-2). Other factors reported to be closely associated with the pathogenesis of AS include matrix metalloproteinase (MMP)-3 and cross-linked telopeptide of type II collagen (CTX-II), which have been recommended as predictors for AS. Serum metalloproteinase MMP-3 has been shown to be effective for predicting AS progression (14) and urinary CTX-II is an important index of cartilage degradation (15). The above biomarkers may provide information that promotes understanding of the prognosis, disease activity, and pathogenesis of AS (16). Thus, these biomarkers are important indexes when evaluating the efficacy of drugs in treating AS.

Tripterygium glycosides tablet (TGTs), is a product of a Traditional Chinese Medicinal plant comprising of triptolides, which have been used in China for the long-term treatment of inflammatory conditions such as rheumatoid arthritis, various skin disorders, chronic nephritis and AS. In recent years, TGT has been used to treat patients with active AS with improved efficiency. However, the mechanism is far from understood.

The aim of the current study was to explore the effects of TGT in the treatment of active AS and identify the possible mechanisms. A total of 36 patients with active AS (AS group) were enrolled and treated with TGT ( $20 \mathrm{mg}, 3$ times/day) for 12 weeks, while 21 healthy, age- and gender-matched volunteers were used as a control group. The levels of several serum biomarkers (DKK-1, IL-17, RANKL, OPG, BAP, CTX-II, MMP-3, PGE2, BMP-2, and VEGF) were assessed in patients with AS prior and subsequent to TGT treatment and in healthy controls.

\section{Materials and methods}

Study population. Ethical approval was obtained from the Human Research Ethics Committee at the Affiliated Hospital of Nanjing University of Traditional Chinese Medicine (Nanjing, China), and participants provided written informed consent. TGT (cat. no. 0802102) was purchased from Deeng Pharmaceutical Company (Zhejiang, China). In the study, 36 patients with AS and 21 unrelated healthy controls who were age- and gender-matched were recruited at the Affiliated Hospital of Nanjing University of Traditional Chinese Medicine between January 2010 and January 2013. All patients and controls were of Han Chinese origin. All the patients with AS were treated with $20 \mathrm{mg}$ TGT three times per day for 12 weeks; no other treatments were given to the patients. The diagnosis of AS was been made by experienced rheumatologists; all diagnoses satisfy the modified New York criteria (17). Subjects with rheumatoid arthritis, inflammatory bowel disease, psoriasis or other autoimmune diseases were excluded from the AS and the control groups. Subjects with additional use of nonsteroidal anti-inflammatory drugs (NSAIDs) or other drugs were excluded from the AS and control groups. The patients were followed-up with for 12 weeks. Peripheral blood samples $(2 \mathrm{ml})$ were obtained from the patients prior and subsequent to TGT treatment for 12 weeks. The samples were collected in heparin-containing tubes (Becton Dickinson, Heidelberg, Germany) and all samples were immediately stored at $-70^{\circ} \mathrm{C}$ until further processing.

Basic data acquisition. The Bath AS disease activity index (BASDAI) was administered to all the patients with AS using questionnaires, as it is the most widely used tool for the assessment of AS functional status and disease activity (18). Serum assays for ESR (erythrocyte sedimentation rate) and CRP (C-reactive protein) were performed on patients with AS prior and subsequent to TGT treatment. ESR was determined using the Westergren method (19) and CRP was determined using an ELISA kit (Kehua Biotech, Shanghai, China). The normal range of ESR is $0-15 \mathrm{~mm} / \mathrm{h}$ and the normal range of CRP is $0-10 \mathrm{mg} / \mathrm{l}$.

\section{Serum dickkopf homolog 1 (DKK1) and IL-17 levels}

Reverse transcription-polymerase chain reaction $(R T-P C R)$. Fifteen patients with AS prior and subsequent to TGT treatment were randomly selected for RT-PCR analysis. The expression levels of DKK1 and IL-17 were measured by RT-qPCR. Total RNA was extracted from serum using TRIzol $^{\circledR}$ (Invitrogen, Carlsbad, CA, USA) according to the manufacturer's instructions. Primer sequences (Sangon Biotech, Shanghai, China) and RT-PCR product length of the investigated factors were listed as follows: Forward, 5'-CCA ACGCTATCAAGAACCTGC-3', and reverse, 5'-TGACCG GAGACAAACAGAACC-3' for DKK1 (461 bp); forward, 5'-ACAACCGATCCACCTCACC-3', and reverse, 5'-CAGCCC ACGGACACCAGTA-3' for IL-17 (232 bp); forward, 5'-AAG GTCGGAGTCAACGGATTT-3', and reverse, 5'-AGATGA TGACCCTTTTGGCTC-3' for human (h)GAPDH (352 bp). cDNA was amplified by PCR in a thermal cycler (Eppendorf, Hamburg, Germany). Thirty-two cycles were performed for hGAPDH and the other primer pairs. The PCR steps were as follows: 1 cycle at $95^{\circ} \mathrm{C}$ for $30 \mathrm{sec}$ for initial denaturation; folllowed by 40 cycles at $95^{\circ} \mathrm{C}$ for $3 \mathrm{sec}$ and $60^{\circ} \mathrm{C}$ for $30 \mathrm{sec}$.

Amplification products were analyzed by electrophoresis and the intensity of the PCR products was quantified using Quantity One software (Bio-Rad, Hercules, CA, USA).

ELISA assay. The activity levels of serum DKK1 and IL-17 were measured using a commercial human DKK1 ELISA kit (Hufeng Biotechnology, Shanghai, China) and a human IL-17 ELISA kit (eBioscience, San Diego, CA, USA) according to the manufacturer's instructions.

Levels of biomarkers in serum (RANKL, OPG, BAP, CTX-II, $M M P-3, P G E 2, B M P-2$ and $V E G F)$. Serum levels of RANKL, OPG, BAP, CTX-II, MMP-3, PGE2, BMP-2 and VEGF were measured using commercial human ELISA kits according to the manufacturer's instructions (RANKL, OPG, BAP and CTX-II from Hufeng Biotechnology; MMP-3, PGE2, BMP-2 and VEGF from KeyGen Biotechnology, Nanjing, China). The serum levels of these factors in the AS group prior or subsequent to TGT treatment were compared with the control group. 
Table I. BASDAI, ESR and CRP for patients with AS before and after TGT treatment.

\begin{tabular}{lcc}
\hline Basic index & Before TGT treatment & After TGT treatmen \\
\hline BASDAI $(\mathrm{mm})$ & $51.94 \pm 11.57$ & $24.47 \pm 13.01^{\mathrm{a}}$ \\
ESR $(\mathrm{mm} / \mathrm{h})$ & $31.18 \pm 15.63$ & $15.87 \pm 14.65^{\mathrm{a}}$ \\
CRP $(\mathrm{mg} / \mathrm{l})$ & $16.30 \pm 11.28$ & $6.10 \pm 6.88^{\mathrm{a}}$
\end{tabular}

${ }^{a} \mathrm{P}<0.05$ compared with the AS group prior to TGT treatment. $\mathrm{N}=36$. BASDAI, Bath AS disease activity index; ESR, erythrocyte sedimentation rate; CRP, C-reactive protein; AS, ankylosing spondylitis; TGT, tripterygium glycosides tablet.
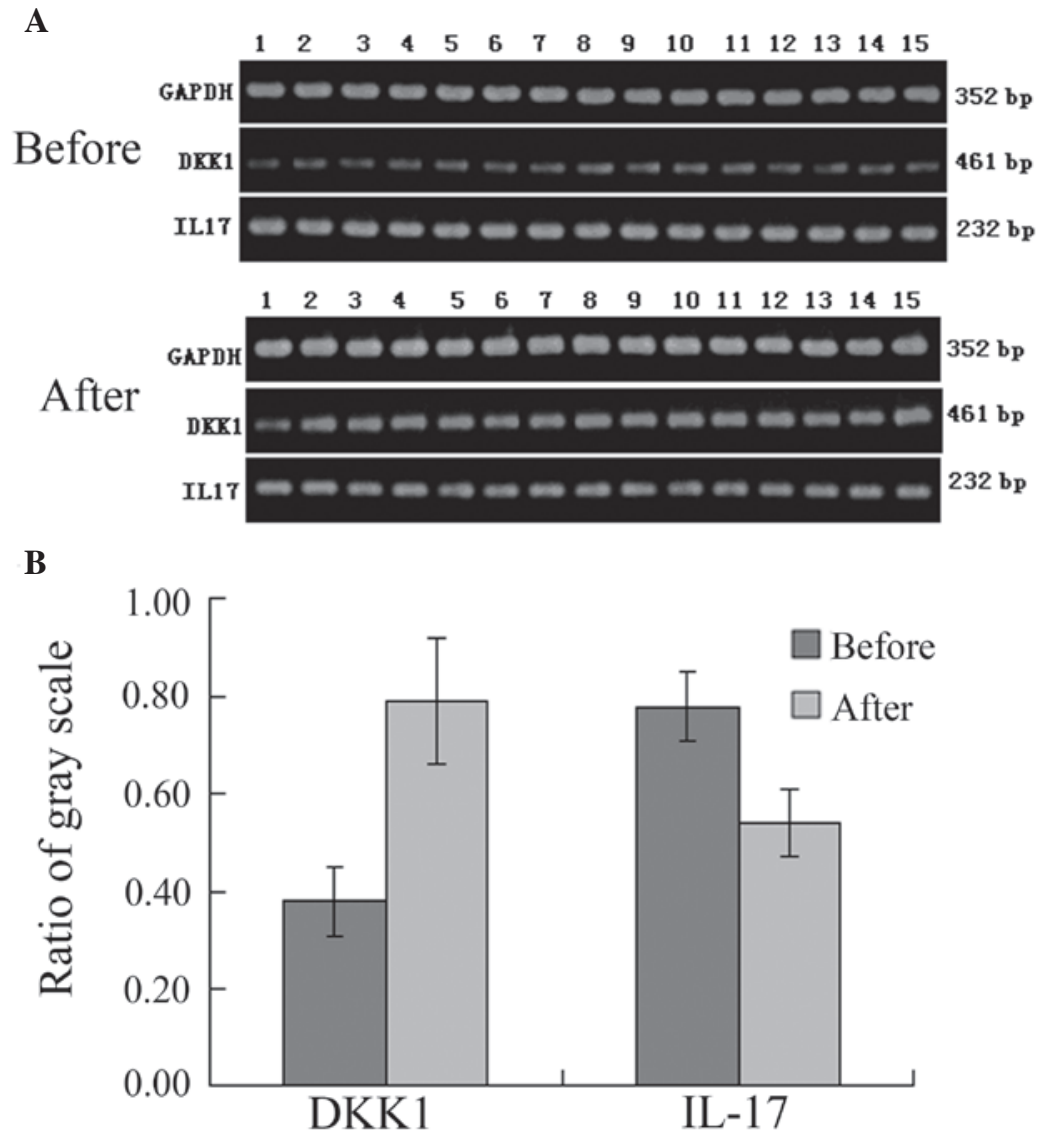

Figure 1. mRNA expression levels of DKK1 and IL-17 in 15 patients with ankylosing spondylitis prior and subsequent to TGT treatment. (A) RT-PCR analysis and (B) grayscale quantification of the RT-PCR analysis ("P<0.05 vs. before TGT treatment). TGT, tripterygium glycosides tablet; RT-PCR, reverse transcription-polymerase chain reaction; DKK1, serum dickkopf homolog 1; IL-17, interleukin-17.

Statistical analysis. Statistical analyses were performed using the statistical software Statistical Packages for Social Sciences (SPSS) version 14.0 (SPSS, Inc., Chicago, IL, USA). Results are expressed as the mean \pm standard deviation. $\mathrm{P}<0.05$ was considered to indicate a statistically significant difference.

\section{Results}

Clinical features. The BASDAI, ESR and CRP of the patients with AS prior and subsequent to TGT treatment are recorded in Table I. Prior to TGT treatment, the BASDAI score in 20 cases was $>50 \mathrm{~mm}$, and average score of BASDAI of all the patients with AS $(51.94 \pm 11.57)$ was higher than normal range. After 12 weeks of TGT treatment, the BASDAI score was significantly reduced $(24.47 \pm 13.01)$. Prior to treatment, the level of ESR and CRP were higher than those of the normal controls. After 12 weeks of TGT treatment, the levels of ESR and CRP were significantly decreased to normal range.

DKKI and IL-17 RNA level in serum. The RNA levels of $D K K 1$ and $I L-17$ in serum of the groups are presented in Fig. 1, which shows the increased DKK1 RNA expression and decreased IL-17 RNA expression following TGT treatment for patients with AS.

DKK1 and IL-17 protein level in serum. The protein levels of DKK1 and IL-17 in serum of the groups are shown in Fig. 2. Prior to TGT treatment, there was a significant reduction in DKK1 expression $(\mathrm{P}<0.05)$ and significant increase in IL-17 expression $(\mathrm{P}<0.05)$ in the AS group compared with the control 

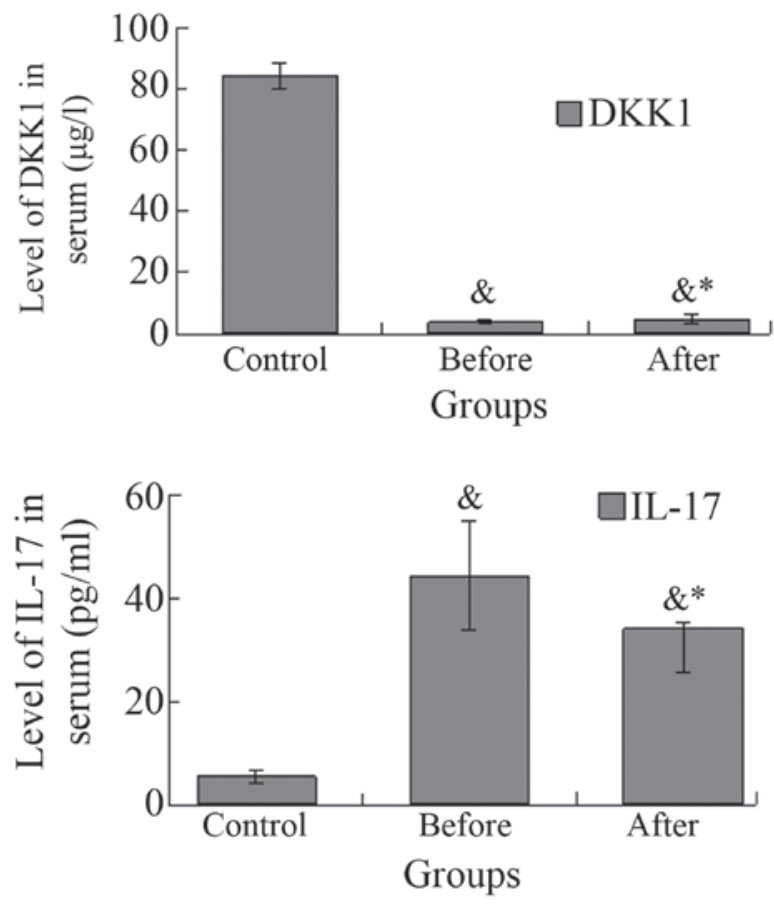

Figure 2. Protein expression of DKK1 and IL-17 in patients with AS prior and subsequent to TGT treatment. "P $<0.05$ compared with the AS group before TGT treatment; ${ }^{\&} \mathrm{P}<0.05$ compared with the control group. AS, ankylosing spondylitis; TGT, tripterygium glycosides tablet; DKK1, serum dickkoph homolog 1; IL-17, interleukin-17.

group. Following TGT treatment for six weeks, the serum DKK1 level in patients with AS were significantly upregulated $(\mathrm{P}<0.05)$, and the serum IL-17 protein level in patients with AS was significantly downregulated $(\mathrm{P}<0.05)$.

Levels of biomarkers in serum (RANKL, OPG, BAP, CTX-II, $M M P-3, P G E 2, B M P-2$ and VEGF). The expression levels of RANKL, OPG, BAP, CTX-II, MMP-3, PGE2, BMP-2, and VEGF in patients with AS prior and subsequent to TGT treatment are shown in Fig. 3. A reduction in serum OPG, BAP and BMP-2 levels were observed in patients with AS compared with those of the control subjects, and increased serum RANKL, CTX-II, MMP-3, PGE2, and VEGF levels were observed in patients with AS compared with those of the control subjects. Following TGT treatment in patients with AS, there was a significant increase in the expression of OPG, BAP and BMP-2 (all P<0.01), and a significant decrease in the expression of RANKL, CTX-II. MMP-3, PGE2 and VEGF $(\mathrm{P}<0.01, \mathrm{P}<0.01, \mathrm{P}<0.01, \mathrm{P}<0.05$ and $\mathrm{P}<0.01$, respectively).

\section{Discussion}

AS is a chronic inflammation of the sacroiliac joints, spine and peripheral joints. NSAIDs are the first stage of medication in treating the pain and stiffness associated with AS. However, NSAIDs have significant side effects, in particular, damage to the gastrointestinal tract. When NSAID treatment is insufficient, second line medications, sometimes referred to as disease modifying anti-rheumatic drugs, are used, including Sulfasalazine and Methotrexate. Tumor necrosis factor (TNF) blockers are one of the most promising medications for treating AS. However, TNF blockers may cause serious side effects, including reactivating latent tuberculosis and neurological problems.

TGT is a product of a Traditional Chinese Medicinal plant that has been used in China for the long-term treatment of inflammatory conditions including rheumatoid arthritis, various skin disorders, chronic nephritis and AS. More recently, TGT has been used to treat patients with active AS and find an improved efficiency. However, the mechanism of action is unclear. In the present study, the expression levels of biomarkers were examined in patients with AS prior and subsequent to TGT treatment, and the possible mechanisms of TGT in the treatment of AS were revealed. Firstly, we investigated the anti-inflammatory effect of TGT on patients with AS. The inflammation caused by AS is associated with the pathogenetic condition and prognosis of AS, that is, patients with AS with higher inflammation level may have worse functional status. Cansu et al (20) found that patients with AS with higher ESR or CRP levels had higher BASFI scores, and that the levels of CRP were significantly associated with a high BASFI. In the present study, we found an elevated ESR level, CRP level and BASDAI score in patients with AS compared with those of the healthy controls, which is consistent with published results. Following TGT treatment, it was shown that the BASDAI score and the ESR and CRP levels of the patients with AS were significantly reduced to a normal level (all $\mathrm{P}<0.05$ ), demonstrating the possible anti-inflammatory effect of TGT on AS. Furthermore, significantly higher serum levels of IL-17, VEGF and PGE2 were observed in patients with AS compared with those of the control subjects (all $\mathrm{P}<0.05$ ), and the levels were revealed to be significantly reduced following TGT treatment for 12 weeks $(\mathrm{P}<0.01, \mathrm{P}<0.01$ and $\mathrm{P}<0.05$, respectively). The results demonstrated that TGT may regulate inflammatory factors, such as IL-17, and inhibit the production of PGE2, which leads to an anti-inflammatory effect on AS. The 

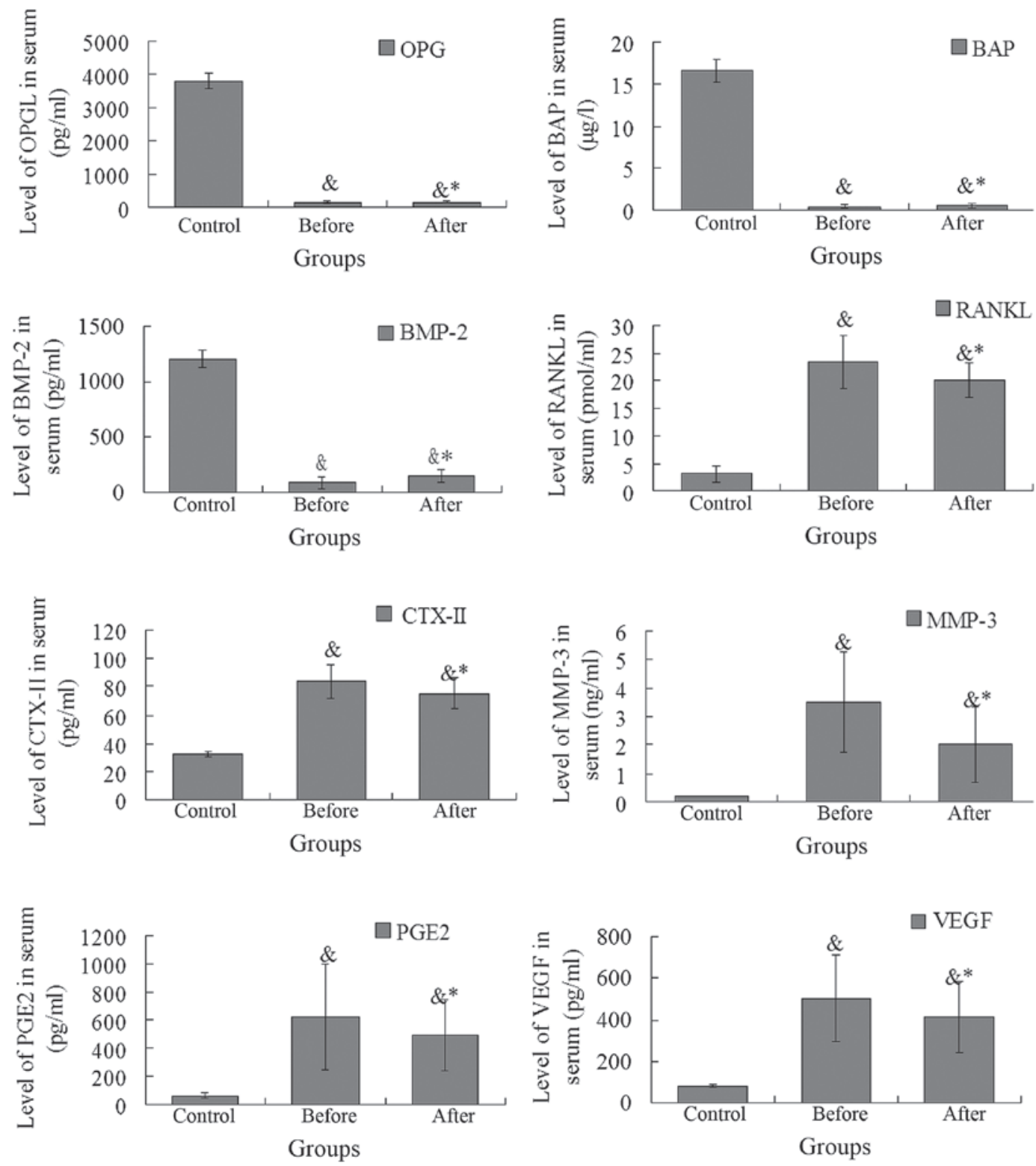

Figure 3. Expression of receptor activator of osteoprotegerin (OPG), bone alkaline phosphatase (BAP), bone morphogenetic protein-2 (BMP-2), nuclear factor $\kappa \mathrm{B}$ ligand (RANKL), cross-linked telopeptide of type II collagen (CTX-II), matrix metalloproteinase-3 (MMP-3), prostaglandin E2 (PGE2) and vascular endothelial growth factor (VEGF) in the control and ankylosing spondylitis (AS) groups before and after tripterygium glycoside (TGT) treatment. ${ }^{*} \mathrm{P}<0.05$ compared with the the AS group before TGT treatment; ${ }^{\&} \mathrm{P}<0.05$ compared with the control group.

changes in the levels of inflammatory cytokines in the serum further demonstrated the possible anti-inflammatory effect of TGT on AS.

Additionally, it was determined that TGT may be a potent regulator of new bone formation in patients with AS. The inflammation caused by rheumatoid arthritis (RA) induces bone destruction, while inflammation caused by AS may induce bone destruction and new bone formation. Bone destruction is associated with large amounts of new bone formation in patients with AS. This is because bone repair occurs after bone destruction, however, while acute lesions resolve completely, more advanced lesions are associated with new bone formation, characterized by fusion of the spine and joints such as sacroiliac joint, clinical stiffness and formation of osteophyma (21). Therefore, inhibiting new bone formation is another potential strategy for the treatment of AS.
Prostaglandins (PGE), bone morphogenetic protein (BMP) and Wnt proteins are the primary factors with essential roles in this bone formation process. PGE2, which has anabolic effects on bones and promotes proliferation, is an important factor for osteoblast differentiation in AS; inducing the expression of bone sialoprotein and bone alkaline phosphatase (BAP) (12). PGE2 synergizes with BMP-2, a member of the transforming growth factor/BMP protein family in inducing bone formation (22). In addition, Wnt proteins have been identified as potent inducers of bone regeneration (23). On the basis of inhibiting new bone formation, the mechanisms of current drugs for treating AS are as follows: i) TNF blockers. TNF induces the expression of Wnt, so TNF blockers improve the signs and symptoms of AS, but can not change radiographic progression. ii) Dkk-1 inducers. Dkk-1 is an inhibitory molecule that regulates the Wnt pathway, which controls osteoblastogenesis, 
and the potential role of Dkk-1 in AS has been explored (13). The Wnt signaling pathway is inhibited by inducing DKK1, thus the new bone formation is inhibited. iii) PGE2 inhibitors. PGE2 downregulates Dkk-1 and sclerostin to increase the activity of the Wnt pathway, thus lead to the new bone formation. By inhibiting the expression of PGE2, certain drugs have shown great efficacy for treating AS, for example NSAIDs. In the present study, significantly lower serum DKK1 levels (RNA/protein) were observed in patients with AS compared with those of the healthy controls which is consistent with the results of Diarra et al (13), in addition to significantly higher serum PGE2 levels. Following TGT treatment for 12 weeks, the Dkk-1 levels (RNA/protein) were significantly upregulated and the PGE2 levels were significant downregulated in patients with AS, demonstrating that TGT may be a potent inhibitor of new bone formation in patients with AS. However, the BMP-2 level in patients with AS was observed to be significantly increased following TGT treatment. We speculate that this is because BMP-2 is regulated by additional signaling pathways, such as intracellular Smad signaling (24). The efficiency of TGT at regulating BMP-2 requires further study.

Thirdly, it was determined that the efficacy of TGT in the treatment of AS may be associated with its bone protective effect. A number of bone metabolism-related factors are also involved in the formation of AS. RANKL, a novel member of the tumor-necrosis factor family, has a key role in osteoclast formation and activation. RANKL is expressed by osteoblast lineage cells and binds with two different receptors, the first of which is OPG, and the second is RANK, which is expressed on the surface of osteoclast lineage cells. The binding of RANKL and RANK on osteoclasts or osteoclast precursor cell activates the transcription factor NF-kB and protein kinase JNK, which promote osteoclast proliferation, differentiation, maturation and bone resorption activity (25). OPG, belonging to the TNF receptor superfamily, is the natural antagonist of RANKL receptor, blocking the linkage of RANKL and RANK, thereby inhibiting the biological effects of RANKL, including such as bone resorption, osteoclast differentiation, activation and induction of apoptosis. OPG is reported to be associated with poor physical mobility and to reflect systemic inflammation in AS (26). Therefore, RANKL promotes osteoclast genesis, while OPG prevents bone erosion (27). In the present study, a significantly lower OPG level and a higher RANKL level were observed in patients with AS compared with those of control subjects. Following treatment with TGT, a significant increase in the OPG level and a reduction in the RANKL level were observed in patients with AS, which shows the potential bone protective effect of TGT by regulating RANKL/OPG signal pathway in patients with AS.

To improve understanding of the progression of AS and the effects of TGT on treating AS, several other serum biomarkers for evaluating the AS progression were measured in the study, including BAP, MMP-3 and CTX-II. BAP, the bone-specific isoform of alkaline phosphatase, has been shown to be a sensitive and reliable indicator of bone metabolism (28). In the current study, a reduction in the serum BAP level was observed in patients with AS compared with that of control subjects, and a significantly increased serum BAP level was found in patients with AS following treatment with TGT. Furthermore, the results revealed significantly higher serum levels of
MMP-3, CTX-II and VEGF in patients with AS compared with control subjects, and the levels were significantly reduced following treatment with TGT. Serum MMP-3 has been shown to be an effective predictor of AS progression (14) and urinary CTX-II is an important index of cartilage degradation (15). Patients with AS have been reported to have significantly higher mean urinary and serum CTX-II levels compared with those of control subjects $(29,30)$, which is consistent with the present results. In conclusion, TGT is effective at improving the signs and symptoms of patients with AS, and the changes in serum biomarkers demonstrate the mechanisms that may be associated with the anti-inflammatory effect, inhibition of new bone formation and a potential bone protective effect.

\section{Acknowledgements}

This study was supported by grants from the Fund for the Talents in Traditional Chinese Medicine of Jiangsu Province (no. LJ200907) and Jiangsu Province Administration of Traditional Chinese Medicine (no. LZ11014). The authors would like to thank Professor David Yu (Medical Center at the University of California, Los Angeles, Doctor of Medicine and Lifetime Professor) for his technical support and suggestions.

\section{References}

1. Rolle AS, Zimmermann B and Poon SH: Etanercept-induced Henoch-Schönlein purpura in a patient with ankylosing spondylitis. J Clin Rheumatol 19: 90-93, 2013.

2. Braun J and Sieper J: Ankylosing spondylitis. Lancet 369: 1379-1390, 2007

3. Calin A, Brophy S and Blake D: Impact of sex on inheritance of ankylosing spondylitis: a cohort study. Lancet 354: 1687-1690, 1999.

4. Ng SC, Liao Z, Yu DT, et al: Epidemiology of spondyloarthritis in the people's republic of china: review of the literature and commentary. Semin Arthritis Rheum 37: 39-47, 2007.

5. Rubin LA, Amos CI, Wade JA, et al: Investigating the genetic basis for ankylosing spondylitis: linkage studies with the major histocompatibility complex region. Arthritis Rheum 37: 1212-1220, 1994.

6. Zeng QY, Chen R and Darmawan J: Rheumatic diseases In China. Arthritis Res Ther 10: 17, 2008.

7. Zochling J and Smith EU: Sero negative spondyloarthritis. Best Pract Res Clin Rheumatol 24: 747-756, 2010.

8. Reveille JD: Epidemiology of spondyloarthritis in North America. Am J Med Sci 341: 284-286, 2011.

9. Wendling D, Cedoz JP, Racadot E, et al: Serum IL-17, BMP-7 and bone turnover markers in patients with ankylosing spondylitis. Joint Bone Spine 74: 304-305, 2007.

10. Mei Y, Pan F, Gao J, et al: Increased serum IL-17 and IL-23 in the patient with ankylosing spondylitis. Clin Rheumatol 30: 269-273, 2011.

11. Goldberger C, Dulak J, Duftner C, et al: Vascular endothelial growth factor (VEGF) in ankylosing spondylitis-a pilot study. Wien Med Wochenschr 152: 223-225, 2002.

12. Samoto H, Shimizu E, Matsuda-Honjyo Y, et al: Prostaglandin E2 stimulates bone sialoprotein (BSP) expression through cAMP and fibroblast growth factor 2 response elements in the proximal promoter of the rat BSP gene. J Biol Chem 278: 28659-28667, 2003.

13. Daoussis D, Liossis SN, Solomou EE, et al: Evidence that DKK-1 is dysfunctional in ankylosing spondylitis. Arthritis Rheum 62: $150-158,2010$.

14. Maksvmowych WP, Landewé R, Conner-Spady B, et al: Serum matrix metalloproteinase 3 is an independent predictor of structural damage progression in patients with ankylosing spondylitis. Arthritis Rheum 56: 1846-1853, 2007.

15. Christgau S, Garnero P, Fledelius C, et al: Collagen type II C-telopeptide fragments as an index of cartilage degradation. Bone 29: 209-215, 2001. 
16. Maksvmowych WP: What do biomarkers tell us about the pathogenesis of ankylosing spondylitis? Arthritis Res Ther 11: $101,2009$.

17. van der Linden S, Valkenburg HA and Cats A: Evaluation of diagnostic criteria for ankylosing spondylitis. A proposal for modification of the New York criteria. Arthritis Rheum 27: 361-368, 1984.

18. Garrett S, Jenkinson T, Whitelock HC, et al: A new approach to defining disease status in AS: the bath ankylosing spondylitis disease activity index. J Rheumatol 21: 2286-2291, 1994.

19. Westergren A: Studies of the suspension stability of the blood in pulmonary tuberculosis. Acta Med Scand 54: 247-282, 1921.

20. Cansu DU, Calışır C, Savaş Yavaş U, et al: Predictors of radiographic severity and functional disability in Turkish patients with ankylosing spondylitis. Clin Rheumatol 30: 557-562, 2011.

21. Maksymowych WP, Morency N, Conner-Spady B, et al: Suppression of inflammation and effects on new bone formation in ankylosing spondylitis: evidence for a window of opportunity in disease modification. Ann Rheum Dis 72: 23-28, 2013.

22. Zhang X, Schwarz EM, Young DA, et al: Cyclooxygenase-2 regulates mesenchymal cell differentiation into the osteoblast lineage and is critically involved in bone repair. J Clin Invest 109: 1405-1415, 2002

23. Minear S, Leucht $\mathrm{P}$, Jiang $\mathrm{J}$, et al: Wnt proteins promote bone regeneration. Sci Transl Med 2: 29-30, 2010.
24. Lories RJ, Daans M, Derese I, et al: Noggin haploinsufficiency differentially affects tissue responses in destructive and remodeling arthritis. Arthritis Rheum 54: 1736-1746, 2006.

25. O'Gradaigh D, Ireland D, Bord S, et al: Joint erosion in rheumatoid arthritis: interactions between tumour necrosis factor $\alpha$, interleukin 1 and receptor activator of nuclear factor $\kappa b$ ligand (RANKl) regulate osteoclasts. Ann Rheum Dis 63: 354-359, 2004.

26. Chen $\mathrm{CH}$, Chen HA, Liao HT, et al: Soluble receptor activator of nuclear factor-kappab ligand (RANKl) and osteoprotegerin in ankylosing spondylitis: OPG is associated with poor physical mobility and reflects systemic inflammation. Clin Rheumatol 29: 1155-1161, 2010.

27. Bao L, Zhu T, Zhao D, et al: Adeno-associated virus-mediated osteoprotegerin gene transfer protects against joint destruction in a collagen-induced arthritis rat model. Joint Bone Spine 79: 482-487, 2012.

28. Kress BC: Bone alkaline phosphatase: methods of quantitation and clinical utility. J Clin Ligand Assay 21: 139-148, 1998.

29. Park MC, Chung SJ, Park YB, et al: Bone and cartilage turnover markers, bone mineral density and radiographic damage in men with ankylosing spondylitis. Yonsei Med J 49: 288-294, 2008.

30. Poddubnyy D, Denis Congad, et al: Predictive and protective value of biomarkers in patients with ankylosing spondylitis who are at high risk of radiographic spinal progression. Arthritis Rheum 63: 1338, 2011. 